\title{
PREVALENCE OF TRANSFUSION-TRANSMITTED INFECTIONS IN BLOOD DONORS IN A TERTIARY CARE CENTRE
}

\author{
Kala V. L1, Kumari K. C. Usha², Prema N. S3, Sajith V4 \\ ${ }_{1}^{1}$ Assistant Professor, Department of Transfusion Medicine, Government Medical College, Kottayam, Kerala. \\ ${ }^{2}$ Professor, Department of Transfusion Medicine, Sree Mookambika Institute of Medical Sciences, Kulasekharam. \\ ${ }^{3}$ Additional Professor, Department of Pathology, Government Medical College, Kollam, Kerala. \\ ${ }^{4}$ Assistant Professor, Department of Transfusion Medicine, Government Medical College, Thrissur, Kerala.
}

\begin{abstract}
BACKGROUND

At present, the number of transfusions approaches 16 million annually. ${ }^{1}$ Transfusions are lifesaving, but they are also associated with life threatening hazards. Prevalence of infection which can be transmitted by transfusion is increasing in the community. In 1991, with every unit of blood there was 1\% chance of transfusion associated problems including Transfusion-transmitted diseases. ${ }^{2}$ Dreadful and life-threatening complications of transfusion are Hepatitis B, Hepatitis C and HIV 1 and 2 . HIV -2 was first confirmed in West Africa. ${ }^{3}$ HIV-1 is the major infection in India, Africa and other developing countries. Usually mandatory tests for five infections are done on blood before transfusion as instructed by National Aids Control Society of India. They are ELISA test for Hepatitis B, Hepatitis C \& HIV (Anti HIV 1 \& 2), VDRL test for syphilis, Antigen detecting card test for Malaria.
\end{abstract}

\section{MATERIALS AND METHODS}

The seroprevalence of five transfusion-transmitted infections i.e. Hepatitis B, C HIV Malaria and Syphilis among blood donors who attended the Department of Transfusion Medicine, Government Medical College, Thiruvananthapuram from November 2007 October 2010 was retrospectively analysed. A Retrospective descriptive study was done. The data of 74280 donors were analysed. The data of the variables were collected from the donor's registration card in the department, variables were collected from the donor's registration card in the department. Total number of donors and seropositives were categorised according to their age, education, socioeconomic status, gender, and marital status. In each category, the seropositivity is compared with the total number of donors in that particular group. Percentage of positivity is calculated from total positives. The percentage of each disease was then analysed according to age, education, socioeconomic status, gender and marital status.

\section{RESULTS}

In the present study, data of 74280 blood donors were analysed retrospectively. The total number of seropositive donors were 2601. This comes up to $3.5 \%$ of total donor population. The distribution of five infections in seropositive donors were as follows: HBV 1547 (59.5\%), HIV 421 (16.2\%), HCV 407 (15.6\%), VDRL 138 (5.3\%) and malaria 88 (3.4\%). Data According to Age GroupData was analysed according to the age group. It was observed that compared to the total donors the seropositivity was comparatively low in the 18-30 years of age group. It was comparatively high in all the other age groups. These findings were statistically significant, $p$ value $<0.01$. HBV was the predominant infection in all the age groups except 51-60 years where HIV was the predominant infection. According to educational status, donors were grouped into professionals, graduation and above, below graduation and students. Majority of the donors belonged to the below graduation group, professionals were of very low number. Comparing the total donors with seropositivity of that particular groups it was observed that the seropositivity was significantly low in professionals, graduation and above, and in students. The seropositivity was significantly high in the below graduation group, $\mathrm{P}$ value $<0.01$. HBV is the predominant infection in all the groups except professionals, where HIV \& HCV were the predominant infections both having equal prevalence. Considering the analysis according to socioeconomic status, majority of the donors belonged to low socioeconomic class. The seropositivity was significantly high in the low socioeconomic class, low in high and upper classes. These findings were statistically significant, $p$ value $<0.01$. When HIV \& HCV were the predominant infections in the upper class, lower and middle classes showed a maximum prevalence of HBV. The seropositivity when analysed according to the marital status the majority of donor population belonged to the unmarried group, seroprevalence was high in the married and low in the unmarried group. This was statistically significant, $\mathrm{p}$ value $<0.01$. HBV was the predominant infection both in the married and unmarried groups. On gender-wise analysis majority of the blood donors were male. Seropositivity was comparatively high in females and low in males. This was statistically significant, $p$ value $<0.01$. HBV was the predominant infection in both groups.

\section{CONCLUSION}

Study showed that the blood donors were from different groups in the community differing in educational qualification, socioeconomic status, etc. The prevalence of transfusion-transmitted infection also varies with these variables. Prevalence of infection was low in 18-30 years age group, professionals, in groups with high educational level and high socioeconomic class. Recruiting donors from different strata of community by giving incentives should be avoided.

\section{KEYWORDS}

Retrospective Study, Seropositivity, Transfusion-transmitted infections.

HOW TO CITE THIS ARTICLE: Kala VL, Usha KKC, Prema NS, et al. Prevalence of transfusion-transmitted infections in blood donors in a tertiary care centre. J. Evolution Med. Dent. Sci. 2017;6(69):4947-4953, DOI: 10.14260/Jemds/2017/1073 
Financial or Other, Competing Interest: None.

Submission 14-07-2017, Peer Review 15-08-2017,

Acceptance 21-08-2017, Published 28-08-2017.

Corresponding Author:

Dr. Kala V. L,

6A, Cloud 9 Apartments,

Law College Road,

Vanchiyoor, P. O.,

Thiruvananthapuram-695035,

Kerala, India.

E-mail: kilakish@yahoo.co.in

DOI: $10.14260 /$ jemds/2017/1073

\section{(c) $(1) \subseteq$}

\section{BACKGROUND}

Prevalence of infection which can be transmitted by transfusion is increasing in the community. Accurate estimate of the risk of transfusion-transmitted infections is important in order to provide an informed basis for patients and physician to decide on allogeneic transfusion vs. other modalities of treatment. At present, the number of transfusion approaches 16 million annually.(1) Transfusion of blood and its products is an effective way of correcting haematological defects.(4) Transfusions are lifesaving, but they are also associated with life threatening hazards. In 1991, with every unit of blood there was $1 \%$ chance of transfusion associated problems including Transfusiontransmitted diseases.(2) Dreadful and life threatening complications of transfusion are Hepatitis-B, Hepatitis $\mathrm{C}$ and HIV 1 and 2. HIV -2 was first confirmed in West Africa.(3) HIV1 is the major infection in India, Africa and other developing countries.(5,6) Transfusion-transmitted infection is still a major concern to patients, physicians and policy makers. ${ }^{(7)}$

Usually mandatory tests for five infections are done on blood before transfusion as instructed by National Aids Control Society of India. They are ELISA test for Hepatitis B, Hepatitis C \& HIV (Anti HIV 1 \& 2), VDRL test for syphilis. Antigen detecting card test for Malaria studies show that the risk of HIV \& HCV transmission through transfusion is 1 in 2 million after introduction of Minipool NAT (MP NAT). Hepatitis B transmission also decreased due to sensitivity of Anti-HB core Antigen tests.

This study is under taken to know the prevalence of seropositivity among blood donors attending the Department of Transfusion Medicine, Government Medical College, Thiruvananthapuram. Total donations in Kerala were 390000 in 2010 with a seropositivity of $1.1 \%$. In Kerala, at present there are no published reports about the role of education, socioeconomic status and community awareness measures through educational materials on reduction of seropositivity among blood donors. The average donations per year in the Department of Transfusion Medicine, Government Medical College, Thiruvananthapuram is approximately 30,000 . On an average, 375 units of blood and blood products are released from this department every day.

\section{MATERIALS AND METHODS}

The seroprevalence of five transfusion-transmitted infections i.e. Hepatitis B, C, HIV Malaria and Syphilis among blood donors who attended the Department of Transfusion Medicine, Government Medical College, Thiruvananthapuram from November 2007 - October 2010 was retrospectively analysed. A retrospective descriptive study was done. The data of 74280 donors were analysed. The data of the variables were collected from the donor's registration card in the department, Total number of donors and seropositives were categorised according to their age, education, socioeconomic status, gender, and marital status. According to age, donors were classified into four groups: 18-30, 31-40, 41-50 and 51-60. Significance of age group in seropositivity of blood donors were then analysed. Education wise the donors were classified into four categories such as Professionals, graduation and above, below graduation and students. Socioeconomic classification was based on Kuppuswamy's Socioeconomic Status Scale (Appendix no. 1). Students were excluded as their socioeconomic status could not be assessed. Upper middle class and lower middle classes were classified as middle class. The upper lower class and lower classes were classified as lower class. In each category, the seropositivity is compared with the total number of donors in that particular group. Percentage of positivity is calculated from total positives. The percentage of each disease will be analysed according to age, education, socioeconomic status, gender and marital status.

Criteria for exclusion of the donors included age less than 18 years or more than 60 years; body weight less than $60 \mathrm{~kg}$; haemoglobin value less than $12.5 \mathrm{~g} / \mathrm{dL}$; recent history of ill health, blood transfusion, surgery, chronic disease or donation within 3 months. Data generated was coded, entered, validated, and analysed using statistical package for Social Science Version 11. Statistical test done was Z Test for Proportion.

\section{RESULTS}

In the present study, data of 74280 blood donors were analysed retrospectively. The total number of seropositive donors were 2601. This comes up to $3.5 \%$ of total donor population.

\begin{tabular}{|c|c|c|}
\hline Donors & Number & Percentage \\
\hline Positivity & 849 & 3.5 \\
\hline Negativity & 23431 & 96.5 \\
\hline Total & $\mathbf{2 4 2 8 0}$ & $\mathbf{1 0 0 . 0}$ \\
\hline \multicolumn{2}{|c|}{ Table 1. Percentage Distribution of } \\
the Sample According to Positivity
\end{tabular}

The distribution of five infections in seropositive donors were as follows: HBV 1547 (59.5\%), HIV 421 (16.2\%), HCV 407 (15.6\%), VDRL 138 (5.3\%) and malaria 88 (3.4\%).

\begin{tabular}{|c|c|c|}
\hline HBV & 1547 & 59.5 \\
\hline HIV & 421 & 16.2 \\
\hline HCV & 407 & 15.6 \\
\hline VDRL & 138 & 5.3 \\
\hline Malaria & 88 & 3.4 \\
\hline Total & $\mathbf{2 6 0 1}$ & $\mathbf{1 0 0}$ \\
\hline \multicolumn{2}{|c|}{ Table 2. Percentage Distribution of Positivity } \\
\hline
\end{tabular}




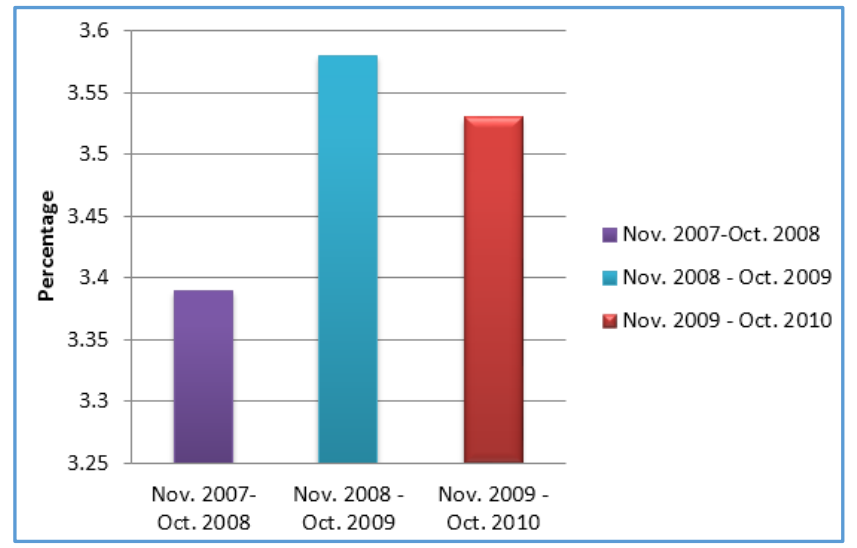

Figure 1. Prevalence of Positivity in Each Year

\section{Data According to Age Group}

Data was analysed according to the age group. The number of blood donors belonging to age group between 18 to 30 years were $49996(67.3 \%)$ among which the total positives were 1482 (57\%). The positivity was considerably lower in this age group, $p$ value of $<0.01$. The percentage of positivity given in the bracket along with the number of positives is the percentage calculated from total positives. When considering the age group 31 to 40 years, the total donors were 19649 (26.5\%) among which 827 (31.8\% of total positives) donors were positive. the positivity was significantly higher considering the other age groups, $\mathrm{P}$ value $<0.01$. In 41 to 50 years of age group, the total of which is 4310 (5.8\%), 193 (7.4\%) were seropositives. The positivity is high in this age group which was statistically significant, $P$ value $<0.01 .51$ to 60 years of age group had a total number of $325(0.4 \%)$ of donors with 99 (3.8\%) seropositives. Here also the positivity was statistically significant, $P$ value $<0.01$.

\section{Data According to Age Group}

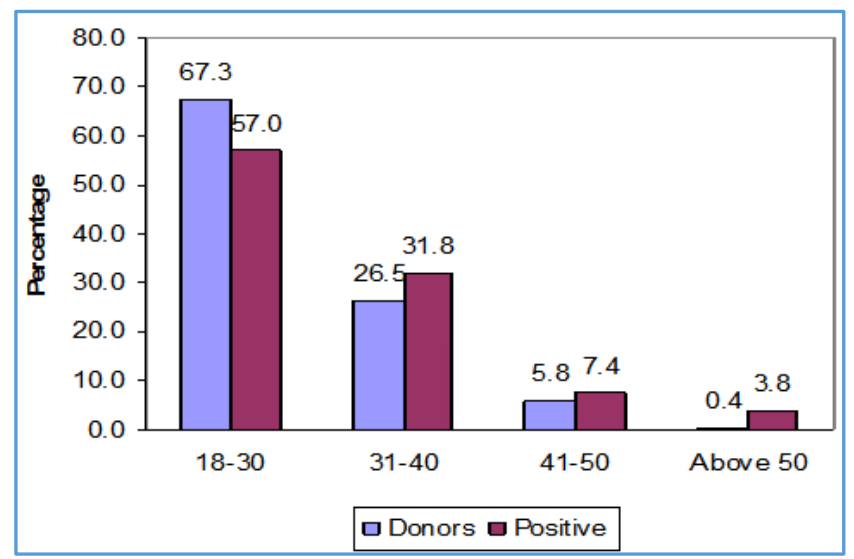

Figure 2. Comparison of Distribution of Donors and Positivity based on Age

The prevalence of positivity based on age group showed 18 to 30 years with $928(62.6 \%)$ of HBV, $236(15.9 \%)$ of HIV, $202(13.6 \%)$ of HCV, 83 (5.6\%) of VDRL and $33(2.2 \%)$ of Malaria. 31 to 40 age group showed 1539 (65.2\%) of HBV, 117(14.1\%) of HIV, 107 (12.9\%) of HCV, 11 (1.3\%) of VDRL and malaria $53(6.4 \%) .41$ to 50 years age group showed a prevalence of $62(65.2 \%) \mathrm{HBV}, 19$ (9.8\%) HIV, 79 (12.9\%) HCV, VDRL 32 (16.6\%) and Malaria 1 (0.5\%). Considering age group 51 to 60 years, number of HBV positives were 18
(18.2\%), HIV 49 (49.5\%), HCV 19 (19.2\%), VDRL 12 (12.1\%) and malaria $1(1 \%)$.

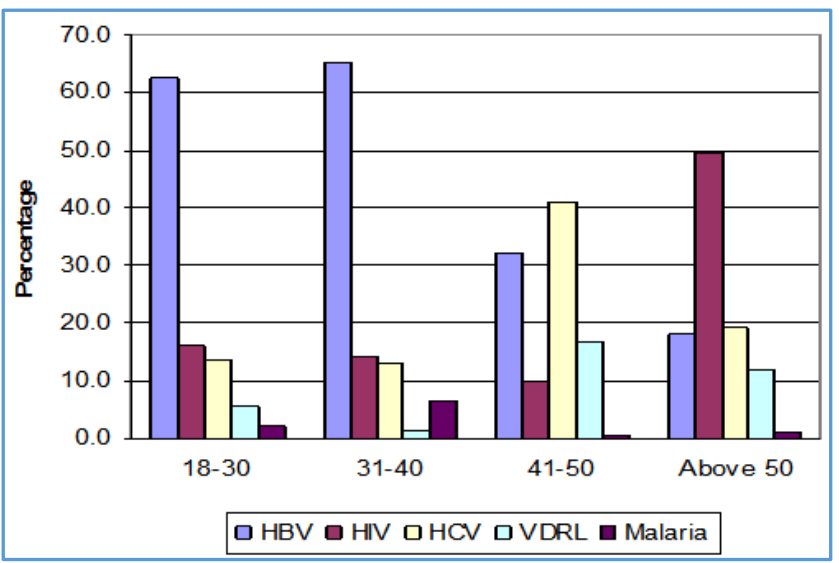

Figure 3. Distribution of Positivity based on Age Group

Considering the age wise prevalence of seropositivity, the most prevalent among all age groups except 51 to 60 years is HBV. In the age group of 51-60 years, HIV is the most prevalent one. Prevalence of malaria is more in 31 to 40 years age group and VDRL is high in 41 to 50 years age group.

\section{Analysis Based on Education}

On analysing the distribution of donors according to educational status, the total number of professionals were $1324(1.8 \%)$ with $22(0.8 \%)$ seropositives. Percentage given in the bracket along with the number of seropositives is the percentage calculated from total positives. Total number of donors with graduation and above is 10956 (4.7\%) with 336 (20.9\%) seropositives. Number of donors in the below graduation group were 46062 (62.0\%) with 1923 (73.9\%) of positives. 15938 students donated blood among which 320 $(12.3 \%)$ were seropositives. The seropositivity is significantly high in the educational group, below graduation and significantly lower in other groups, $\mathrm{P}<0.01$.

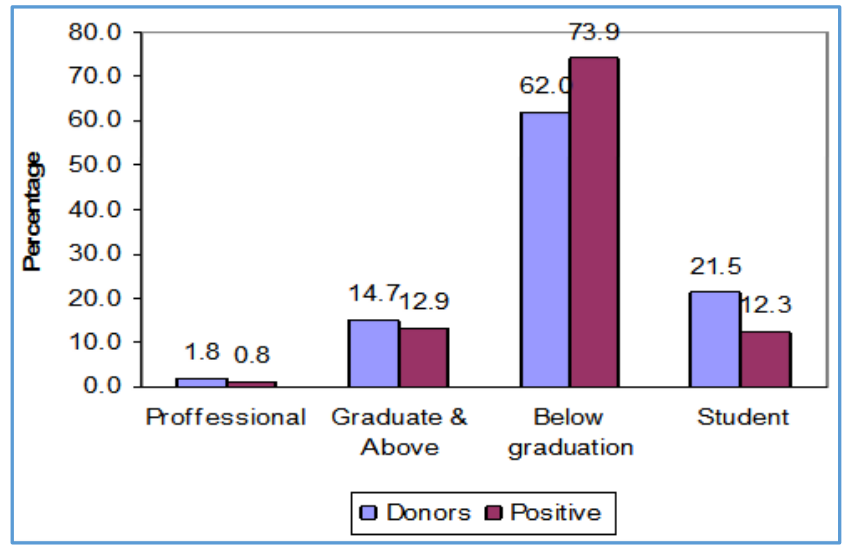

Figure 4. Comparison of Distribution of Donors and Seropositivity based on Education

On analysing the prevalence of the five infections based on education, it was observed that the professionals show only HIV and HCV (50\%) each, the educational group having graduation and above shows HBV 241 (71.7\%), HIV 40 (11.9\%), HCV 33 (9.8\%), VDRL 11 (3.3\%), and Malaria 11 (3.3\%). The prevalence of infection in the below graduation 
group was HBV 1143 (59.4\%), HIV 285 (14.8\%), HCV 321 (16.7\%), VDRL 97 (5\%) and Malaria 77 (4.0\%). In students, the prevalence was as HBV 163 (50.9\%), HIV 85 (26.6\%), HCV 42 (13.1\%), VDRL 30 (9.4\%). No Malarial parasite was detected.

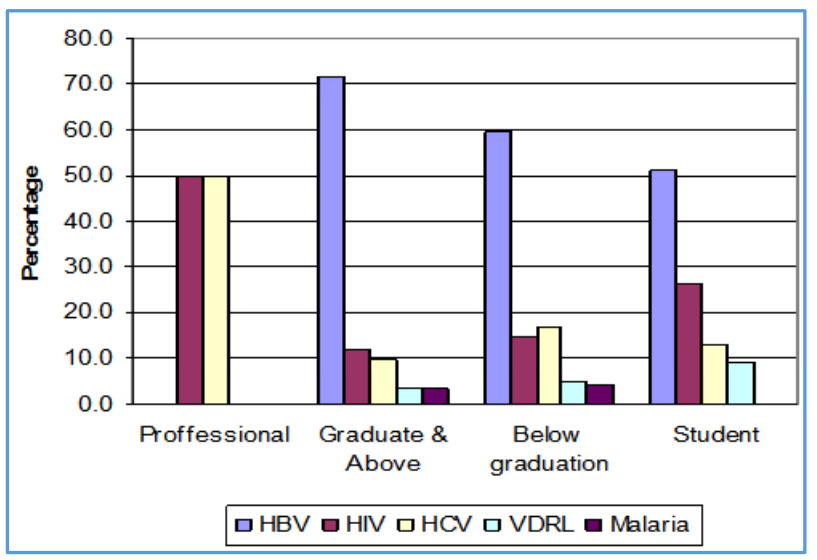

Figure 5. Distribution of Positivity based on Education

Except in professionals the predominant infection in all the other educational groups were HBV. In Professionals, only HIV and HCV were detected and with the same rate of prevalence.

\section{Analysis Based on Socioeconomic Status}

On analysis based on socioeconomic status, students were excluded as their socioeconomic status could not be assessed. Upper class with a total number of 3077 (5.3\%) donors had $22(1.0 \%)$ seropositives. Middle class with 10112 (17.3\%) donors had 215 (9.4\%) seropositives. Among lower class with $45153 \quad(77.4 \%)$ donors, $2044 \quad$ (89.6\%) were seropositives. The seropositivity was significantly low in the upper and middle classes, $P$ value $<0.01$. The seropositivity is significantly high in lower socioeconomic class.

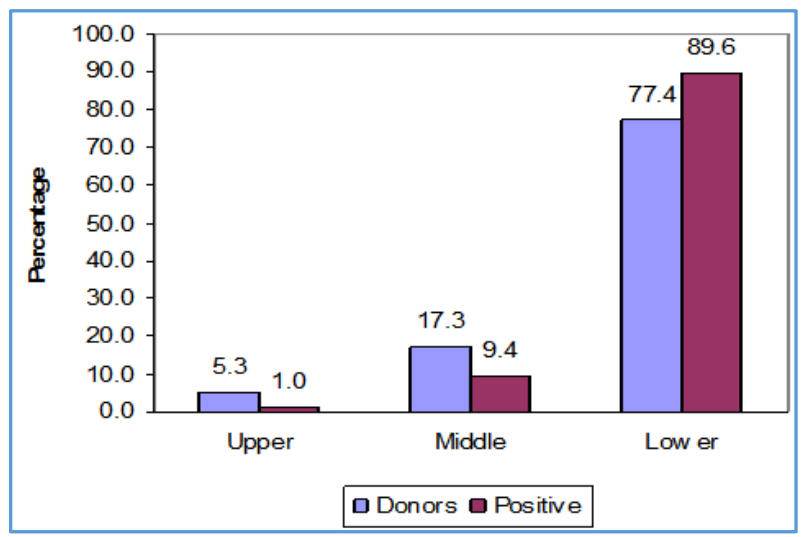

Figure 6. Distribution of Donors and Seropositivity based on Socioeconomic Status

Prevalence of each infection according to socioeconomic status is as follows. In the upper class: HBV 2 (9.1\%) HIV 9(4) 0.9\%, HCV 10 (4) (5.5\%) and VDRL 1 (4).5\% no malarial parasite detected. Middle class: HBV 150 (69.8\%), HIV 20 (9.3\%), HCV 23 (10.7\%), VDRL 21 (9.8\%) and Malaria 1 (0.5\%). Lower class: $1232(60.3 \%)$ HBV cases, $307(15.0 \%)$ HIV cases, 332 (16.2\%) HCV, 36 (4.2\%) VDRL and 37 (4.3\%) Malaria cases.

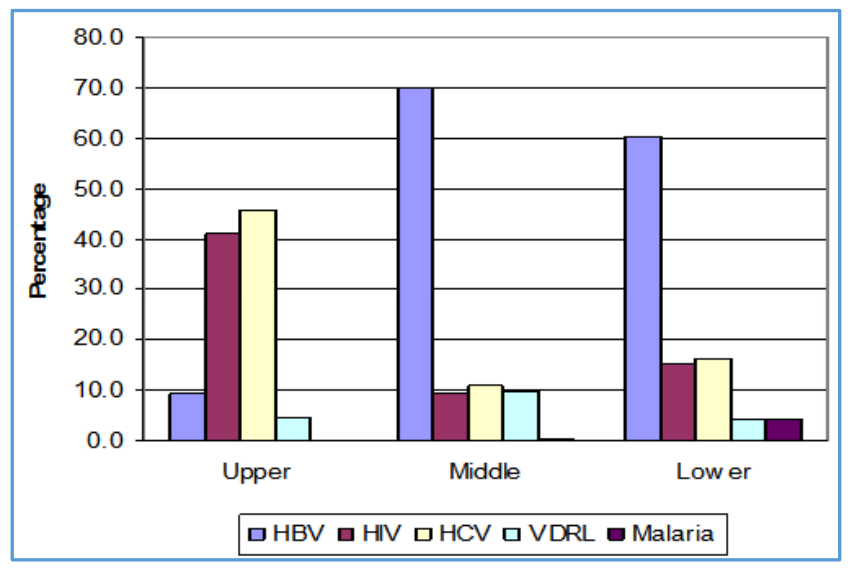

Figure 7. Distribution of Positivity based on Socioeconomic Status

In the upper socioeconomic class, HIV and HCV were the predominant infections. In the middle and lower classes, prevalence was more for HBV.

\section{Analysis Based on Marital Status}

On Analysis based on marital status out of the total 28971 (39\%) of married donors, 1244 (47.8\%) were seropositives. Among 45309 (61.1\%) of unmarried donors, 1357 (52.2\%) were Seropositives. The Seropositivity in the married donors are significantly high compared to that in the unmarried donors which was significantly low, $\mathrm{P}$ value $<0.01$.

\begin{tabular}{|c|c|c|c|c|c|c|}
\hline Marital & \multicolumn{2}{|c|}{ Negative } & Positive & \multicolumn{2}{c|}{ Donors } \\
\cline { 2 - 7 } Status & Count & Percent & Count & Percent & Count & Percent \\
\hline Married & 27727 & 38.7 & 1244 & 47.8 & 28971 & 39.0 \\
\hline Unmarried & 43952 & 61.3 & 1357 & 52.2 & 45309 & 61.0 \\
\hline Total & $\mathbf{7 1 6 7 9}$ & $\mathbf{1 0 0 . 0}$ & $\mathbf{2 6 0 1}$ & $\mathbf{1 0 0 . 0}$ & $\mathbf{7 4 2 8 0}$ & $\mathbf{1 0 0 . 0}$ \\
\hline Table 3. Comparison and Distribution of Donors and \\
Positivity based on Marital Status \\
\hline
\end{tabular}

On analysis of each infection based on marital status, the observation was $723(58.1 \%)$ of HBV in the married donors. HIV was 186 (15\%), HCV 192 (15.4\%), VDRL 66 (5.3\%) and Malaria 77 (6.2\%). In the unmarried group, the prevalence of HBV was 824 (66.7\%), HIV 235 (17.3\%), HCV 215 (15.8\%), VDRL 72 (5.3\%) and Malaria $11(0.8 \%)$

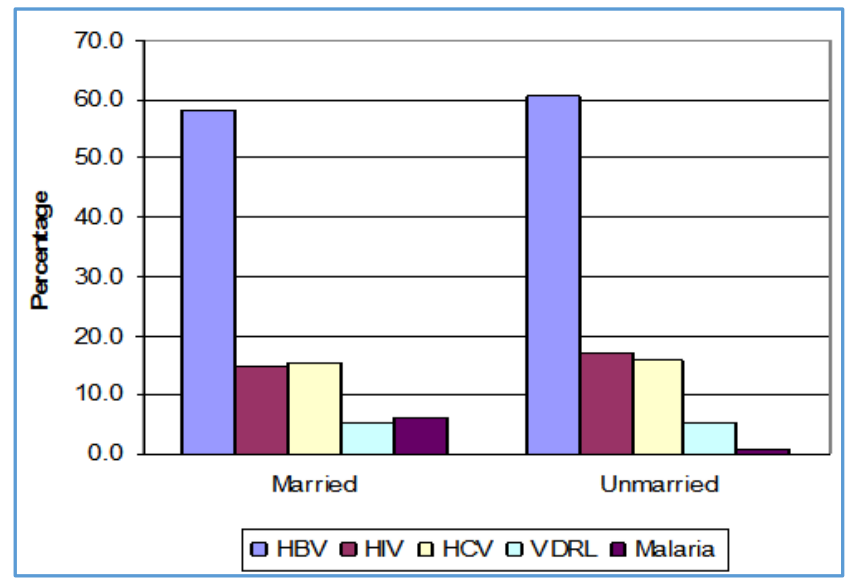

Figure 8. Distribution of Positivity based on Marital Status

In both married and unmarried groups, the most prevalent infection was HB. 
Analysis based on gender

In gender wise classification, total males are 68219 (91.8\%) of which 2284 (87.8\%) are seropositives. Out of total 6061 (8.21\%) females, 317 (12.2\%) are seropositives.

\begin{tabular}{|c|c|c|c|c|c|c|}
\hline \multirow{2}{*}{ Gender } & \multicolumn{2}{|c|}{ Negative } & \multicolumn{2}{c|}{ Positive } & \multicolumn{2}{c|}{ Donors } \\
\cline { 2 - 7 } & Number & Percentage & Number & Percentage & Number & Percentage \\
\hline Male & 65935 & 92.0 & 2284 & 87.8 & 68219 & 91.8 \\
\hline Female & 5744 & 8.0 & 317 & 12.2 & 6061 & 8.2 \\
\hline Total & 71679 & 100.0 & 2601 & 100.0 & 74280 & 100.0 \\
\hline \multicolumn{4}{|r}{ Table 4. Comparison of Distribution of Donors and Positivity based on Gender } \\
\hline
\end{tabular}

P value $<0.01$

\section{Analysis Based on Gender}

On analysing the distribution on gender basis, prevalence of seropositivity in males was as follows: HBV $1373(60.1 \%)$ HIV 344 (15.1\%), HCV 341 (14.9\%), VDRL 138 (6.0\%) and Malaria 88 (3.9\%). In females, HBV was 174 (54.9\%), HIV 77 (24.3\%) and HCV 66 (20.8\%). There were no detected cases of VDRL and Malaria in the females.

\begin{tabular}{|c|c|c|c|c|}
\hline \multirow{2}{*}{} & \multicolumn{2}{|c|}{ Male } & \multicolumn{2}{c|}{ Female } \\
\cline { 2 - 5 } & Number & Percentage & Number & Percentage \\
\hline HBV & 1373 & 60.1 & 174 & 54.9 \\
\hline HIV & 344 & 15.1 & 77 & 24.3 \\
\hline HCV & 341 & 14.9 & 66 & 20.8 \\
\hline VDRL & 138 & 6.0 & 0 & 0.0 \\
\hline Malaria & 88 & 3.9 & 0 & 0.0 \\
\hline Total & $\mathbf{2 2 8 4}$ & $\mathbf{1 0 0 . 0}$ & $\mathbf{3 1 7}$ & $\mathbf{1 0 0 . 0}$ \\
\hline \multicolumn{4}{|c|}{ Table 5. Distribution of Positivity based on Gender } \\
\hline
\end{tabular}

\section{DISCUSSION}

Blood donors belong to heterogeneous groups of people in the society differing in their demographic characteristics and the psychological factors that motivate their behaviour. ${ }^{6}$

In this present study, it is observed that different rates of transfusion-transmitted infection exist in donors belonging to different age groups, educational qualification, socioeconomic status, gender and marital status.

The seropositivity among donors is $3.5 \%$ which is much higher compared to the average in the state which is $1.1 \%$. It is also not in accordance with a previous study conducted in India, where the seroprevalence was $0.6 \% .^{(7)}$

The maximum number of donors were in the age group of $18-30$ years $(67.3 \%)$ followed by $31-40$ years $(26.5 \%)$. The donors of age group 41-50 was only 5.3\% and above 51-60 years was $0.4 \%$.

These findings partly agree with previous study conducted in Nigeria, where majority of donors belonged to 20-29 age group which was $44.5 \%$. Two contradicting reports were obtained from Brazil where the maximum number of donor population was between 50 to 60 years of age.(2)

The positivity among 18-30 years age group was lower (57\%) compared to the percentage of total donors belonging to that age group. This was statistically significant. In 31-40 years age group, the percentage of positivity was higher $(31.8 \%)$ compared to the percentage of donors (26.5\%). This is also statistically significant. Similar findings were seen in age groups 41-50 years and 51-60 years. The percentage of donors were $5.8 \%$ and $0.4 \%$ respectively. The percentage of positivity is $7.4 \%$ and $3.81 \%$ respectively and these findings were also statistically significant.
The findings were in accordance with a previous study in Saudi Arabia where the donor population was high in the 2029 years age group $(50.8 \%)$. The percentage of positivity was comparatively less $(1.4 \%)$ compared to the other groups. The comparative positive was highest above 50 years age group, $2.6 \%$ of donors with $2.6 \%$ of positivity.

This points to the fact that 18-30 years age group included mainly the students who had a higher degree of awareness which reduced the positivity. (8) Psychographics of blood donors having higher income, education and who were in professional donation constituted altruism and selfsacrifice. ${ }^{(9)}$

In this present study, on education wise analysis professionals showed a significant reduction in the positivity. The number of donors were $1.8 \%$ and positives were $0.8 \%$.

The donors with graduation and above showed $14.7 \%$ of donors with $12.9 \%$ of positivity. This is significantly lower. Similar findings are seen in students with $21.5 \%$ total donors and a significantly lower seropositivity (12.3\%). In donors with educational qualification below graduation, the total donors are $62.0 \%$. Compared to that percentage of seropositivity was higher (73.9\%) which was statistically significant.

This points to the fact that positivity was less in students and other educated groups. This highlights the role of education and awareness in reducing disease transmission through transfusion. They can appreciate the health history questionnaire more. They are also more aware about the infectivity and window period of these disease-causing agents. The awareness among the educational group is increased through community programmes and programmes through the media. This points to the importance of recruiting donors from a particular group with less infectivity.(10) Donor recruitment and interviewing reduce the prevalence of infection in donation from first time blood donors by $80 \%$.(11) Repeat and safe blood donors are more among the educated group.(12)

On considering the socioeconomic status of the donors, students were excluded because their socioeconomic status could not be assessed. Majority of donor pool constituted the low socioeconomic class $(77.4 \%)$ followed by middle class $(17.3 \%)$ and then the upper class (5.3\%). Either it may be a reflection of the socioeconomic pattern of the community or the lower socioeconomic class are coming forward for some incentive.(13) Donors of a low socioeconomic status, who had higher rate of transfusion-transmitted infections are attracted by incentives.(13,14) Poverty, lack of modern health care facilities, associated cultural, socioeconomic and values of communities expose individuals to risky behaviour.(15-17) It must be remembered that this being a hospital-based study 
from Government sector, it need not reflect the donor base in the community.

A significantly lower seropositivity was observed among donors of upper and middle class. It was $1.0 \%$ and $9.4 \%$ respectively. Positivity in low socioeconomic class was high i.e. $89.6 \%$. This finding was statistically significant. This finding is consistent with those obtained from studies. Safety and supply of blood are profoundly impacted by the method of recruitment, specific health history taking and blood screening.(12)

When considering the marital status 39\% were married and $61.0 \%$ were unmarried. A significantly higher percentage of seropositivity was noticed among the married $(47.8 \%)$ donors compared to that of the unmarried (52.2\%) donors. This may be due to the fact that the majority of unmarried donors are from the 18-30 years age group and many of them are students.

On a gender based analysis, it was observed that out of the total 74280 donors, 68219 (91.8\%) were male and 6061 $(8.2 \%)$ were female. This finding is consistent with the previous study. ${ }^{(18,19)}$

On considering the seropositivity, it was comparatively higher among females than in the males. The percentage of zero positivity in males is $37.8 \%$ and in females is $12.2 \%$.

This points to the fact that high risk females are coming forward for donation knowingly or unknowingly about their risk status. Another possibility is blood donation for test seeking.(11)

Considering the Prevalence of five mandatory diseases, each was as follows. HBV had the maximum prevalence of $59.5 \%$ followed by HIV and HCV which were $16.2 \%$ and $15.6 \%$ respectively. Prevalence of VDRL was $5.3 \%$ and malaria was $3.4 \%$.

This is partly consistent with the study conducted in July 2010 at which incidence of HBV was the maximum and both HIV and HCV had almost the same prevalence.(7) In Bangalore, the prevalence of HIV and HCV was the same while in Iran HCV had a higher prevalence with decreased prevalence of HIV.(20)

HBV showed maximum prevalence in all age groups except in 51-60 years of age group where HIV was the most prevalent infection. Malaria was more prevalent in 31-40 years age group and VDRL in 41-50 years age group. The prevalence of HIV in 51-60 years age group also points to the fact that people might be coming forward for donation for test seeking.(11)

Considering the educational group in graduation and above, below graduation and students, the predominant infection was HBV unlike in professionals which had a maximum prevalence of HIV and HCV.

Considering the socioeconomic status, HIV and HCV were the predominant infections which was almost the same in the upper socioeconomic class. HBV had high prevalence in the middle and lower socioeconomic classes. Prevalence of VDRL was high in the middle class, where it was almost the same as that of HIV and HCV. Prevalence of malaria was high in the lower socioeconomic class. This finding contradicts a previous study in Pakistan where malarial infection was more in middle class. ${ }^{(21)}$

Hepatitis B transmission is associated with low socioeconomic status education, large family size, crowded poor areas and decreased hygiene.(22) Considering the prevalence of infection according to marital status, there was no significant difference between the married and unmarried. In both maximum prevalence was for HBV.

Considering the gender wise distribution, HBV was prevalent in both males and females. Distribution of the other infections were almost the same in both groups except for malaria and VDRL which were absent in females.

\section{CONCLUSION}

This retrospective study showed that the blood donors were from different groups in the community differing in educational qualification, socioeconomic status, etc. The prevalence of transfusion-transmitted infection also varies with these variables. Prevalence of infection was low in 18-30 years age group, professionals, in groups with high educational level and high socioeconomic class. Recruiting donors from different strata of community by giving incentives should be avoided. Safe donors who donate repeatedly should be promoted. Donor recruitment should be done only from selected groups of donors having high education and socioeconomic level.

\section{REFERENCES}

[1] The United States Department of Health and Human Services. The 2007 National Blood Collection and Utilization Survey Report. Washington, DC: DHIS. 2009.

[2] Widmann FK. Technical manual American association of Blood Banks. $9^{\text {th }}$ edn. Aglington, USA: 1985:325-44.

[3] De Cock KM, Brun-Vezinet F, Soro B. HIV-1 and HIV-2 infections and AIDS is West Africa. AIDS 1991;5(Suppl 1):S21-8.

[4] Garg S, Mathur DR, Garg DK. Comparison of seropositivity of HIV, HBV, HCV and syphilis in replacement and voluntary blood donors in western India. Indian J Pathol Microbiol 2001;44(4):409-12.

[5] Sarin SK, Saxena R, Sundaran KR. Transfusion risks and estimates in Indian population. In: Sarin SK, Hess G. eds. In transfusion associated hepatitis, diagnosis, treatment and prevention. New Delhi: CBS Publications 1998:212-20.

[6] Joshi SR. Seropositive status for HIV infection among voluntary and replacement blood donors in the city of Surat from western Indian. Indian J Hemat and Blood Tranfusion 1998;16(1):20-1.

[7] Fernandez H, D'souza PF, D'souza PM. Prevalence of transfusion-transmitted infections in voluntary and replacement donors. Indian J Hematol Blood Transfusion 2010;26(3):89-91.

[8] Goncalez T, Sabino EC, Chamone DF. Trends in the profile of blood donors at a large blood center in the city of Sao Paulo, Brazil. Rev Panam Salud Publica 2003;13(2-3):144-8.

[9] Korelitz JJ, Williams AE, Busch MP, et al. Demographics characteristics and prevalence of serologic markers among donors who use the confidential unit exclusion process: the retrovirus epidemiology donor study. Transfusion 1994;34(10):870-6.

[10] Oswalt RM. A review of blood donor motivation and recruitment. Transfusion 1977;17(2):123-35. 
[11] Barreto CC, Sabino EC, Goncalez TT, et al. Prevalence, incidence, residual risk of human immunodeficiency virus among community and replacement first time blood donors in Sao Paulo, Brazil. Transfusion 2005;45(11):1709-14.

[12] Rahman M, Akhtar GN, Qadeer M, et al. Safe blood begins with safe donors. Pak J Med Sci 2003;19(3):161-8.

[13] Piliavin JA. Why do they give the gift of life? A review of research on blood donors since 1977. Transfusion 1990;30(5):444-59.

[14] Dawson GJ, Lesniewski RR, Stewart JL, et al. Detection of antibodies to hepatitis C virus in U.S. blood donors. J Clin Microbiol 1991;29(3):551-6.

[15] McQuillan GM, Kruzon-Moran D, Granade T, et al. Seroprevalence of HIV in the U.S household population aged 18-49 years: The National Health And Nutrition Examination Surveys, 1999-2006. J Acquir Immune Defic Syndr 2010;53(1):117-23.

[16] Musbacher J, Chiavetta J, Naiman R, et al. Evaluation of a confidential method of excluding blood donors exposed to human immunodeficiency virus: studies on hepatitis and cytomegalovirus markers. Transfusion 1987;27(2):207-9.
[17] Menitove JE, Lewandowski C, Ashworth LW, et al. Confidential unit exclusion process continues to identify donors with an increased frequency of HIV seropositivity. Transfusion 1991;31:69S.

[18] Mathai J, Sulochana PV, Sathyabhama S, et al. Profile of transfusion transmissible infections and associated risk factors among blood donors of Kerala. Indian J Pathol Microbiol 2002;45(3):319-22.

[19] Olokoba AB, Olokoba LB, Salawu F, et al. Syphilis in voluntary blood donors in North Eastern, Nigeria. European Journal of Scientific Research 2009;31(3):335-40.

[20] Mini SM, Khedmat $H$, Alavine SM, et al. Trends in seroprevalence of hepatitis B, C, HIC, HIV and syphilis in Iranian blood donors-important potential of public benefits. New English J of Medicine 2003;327(6):36973.

[21] Ali N, Ahmed J, Jehan F, et al. Transfusion transmitted malaria in three major blood banks of Peshawar, Pakistan. African Journal of Biotechnology 2010;9(33):5445-9.

[22] Ruff TA, Gertig DM, Otto BF, et al. Lombok hepatitis B model immunization project: toward universal infant and hepatitis B immunization in Indonesia. J Infect Dis 1995;171(2):290-6. 